\title{
Radiative edge cooling experiments in Wendelstein 7-X start-up limiter campaign
}

T. Barbui ${ }^{1}$, F. Effenberg${ }^{1}$, R. König ${ }^{2}$, M. Krychowiak ${ }^{2}$, O. Schmitz ${ }^{1}$ J. Baldzuhn², B. D. Blackwell ${ }^{3}$, B. Buttenschön ${ }^{2}$, P. Drews ${ }^{4}$, M. Jakubowski², S. Kwak², Y. Liang ${ }^{4}$, S. Liu ${ }^{4}$, H. Niemann ${ }^{2}$, T. Sunn Pedersen ${ }^{2}$, J. Svensson ${ }^{2}$, D. Zhang ${ }^{2}$ and the W7-X Team ${ }^{2}$

\author{
${ }^{1}$ Department of Engineering Physics, University of Wisconsin-Madison, Madison, WI 53706, USA \\ ${ }^{2}$ Max-Planck-Institut für Plasmaphysik, 17491 Greifswald, Germany \\ ${ }^{3}$ Plasma Research Laboratory, Research School of Physics and Engineering, Australian National University, Canberra, ACT \\ 0200 Australia \\ ${ }^{4}$ Forschungszentrum Jülich GmbH, Institut für Energie- und Klimaforschung - Plasmaphysik, Partner of the Trilateral Euregio \\ Cluster (TEC), 52425 Jülich, Germany \\ E-mail: barbui@wisc.edu
}

\begin{abstract}
Impurity seeding experiments during the start-up limiter campaign of Wendelstein 7-X provide first evidence for a localization effect of the three-dimensional magnetic edge structure on the seeded impurities and their radiation distribution and cooling effect. Moreover, species dependencies have been seen. Nitrogen was observed to cool the entire edge plasma, with a stronger electron temperature reduction measured at the downstream position at the limiter. The radiation was limited at the periphery of the confined region. Both the temperature reduction and the radiation enhancement were directly correlated to the injection of the coolant gas. Mitigation of the limiter heat loads was also measured. Neon was observed to affect also the confined plasma with a long-lasting radiation and a cooling of the entire plasma.
\end{abstract}

\section{Introduction}

The new optimized helical advanced stellarator device Wendelstein 7-X (W7-X) started its first plasma operation (OP1.1) in a limiter configuration [1][2]. The last closed flux surface (LCFS) of the plasma was defined by 5 poloidal uncooled graphite limiters [3]. In this initial campaign, besides the commissioning of the main device systems and diagnostics required for the next divertor campaign [4][5][6], it was also possible to perform first physics experiments. Among those was the cooling of the edge plasma by impurity seeding of nitrogen $\left(\mathrm{N}_{2}\right)$ and neon $(\mathrm{Ne})$. These experiments aimed to test the method in the specific scrape-off layer geometry of the W7-X limiter campaign and to provide first actual experiments of radiative edge cooling in stellarators in view of the island divertor phase of W7-X. This divertor concept will be used for the future quasi-steady-state operations and it is expected that the heat flux reaching the plasma facing components will be one of the major concerns for the successful operation of the device [7][8]. One possible method to reduce this heat flux is to use intrinsic and actively injected impurities species in the edge of the plasma to dissipate energy by impurity radiation. This method of radiative cooling of the plasma edge by impurity seeding has been extensively used in many devices to remove the heat from the divertor/edge plasma in order to protect the plasma facing components. As radiators, typically low to mid charge state $(Z)$ elements like nitrogen $(\mathrm{N})$, neon $(\mathrm{Ne})$ or argon $(\mathrm{Ar})$ are used. Radiative edge cooling with such gas species has been investigated already in different divertor tokamaks where, besides the purpose of radiative edge cooling, non-intrinsic seeded impurities have also been shown to be effective to mitigate ELMs and to facilitate the detachment [9][10][11][12]. Limiter tokamaks have reached an improved confinement regime through a neon radiative edge [13]. For stellarator devices there is not yet a large experience, and for the island divertor specifically only very limited initial experiments were conducted in the short island divertor campaign at W7-AS. Only in the Large Helical Device (LHD) impurity seeding experiments were carried out. Here, $\mathrm{Ne}, \mathrm{N}_{2}$ and $\mathrm{Kr}$ were used as most effective species and enhancement of plasma radiation as well as reduction of the divertor heat load were observed without significant confinement degradation [14][15]. Ne was observed to radiate considerably from the confined region, although in its periphery, in addition to the strong edge radiation. $\mathrm{N}_{2}$ instead was observed to radiate only from the edge region [16]. 
In this paper, we report on a first-time assessment of radiative edge cooling properties for the limiter start-up scenarios at W7-X. These experiments are unique in the sense that the 3D magnetic edge structure for this campaign was available for the first time in any stellarator device. In particular, the magnetic structure is nicely ordered into three separated magnetic flux bundles, which represent the helical scrape-off layer (SOL) in this configuration [17][18]. This setup hence bears unique opportunities to better understand the generic properties of impurity transport and radiation related to radiative edge cooling. Also, the setup enables for the first time to assess the interaction of a radiative mantle with the helically twisted, close fitting first wall at W7-X, which represents a mixture of first wall materials and might mandate cooling of the plasma boundary layer much before even considering heat flux tolerances for actively cooled components in the divertor. In the experiments considered here, $\mathrm{Ne}$ and $\mathrm{N}_{2}$ were injected through a versatile piezo valve setup [6] located comparably close to the last closed flux surface (LCFS), about $18 \mathrm{~cm}$ away from it. The cooling effects on the plasma edge were measured by Langmuir probes [6] and a new thermal helium beam system [19]. The mitigation of the heat loads on the limiter were assessed by infra-red (IR) imaging spectroscopy of surface temperatures [28]. The poloidal radiated power distribution was measured by a bolometer array [29].

The first goal of these experiments was to demonstrate their feasibility by achieving and measuring the cooling effects. The second goal was to resolve in which region the cooling was dominant (scrape-off layer vs confinement) and to understand whether local or global cooling was predominant in the $3 \mathrm{D}$ scrape-off layer that characterizes the W7-X limiter scenario. These experiments were also useful in preparation for the foreseen feedback-controlled gas injection to be implemented for the next divertor campaigns. Such a system will be based on the control of the edge $T_{\mathrm{e}} / n_{\mathrm{e}}$ or the edge impurity radiation by flow-controlled gas injection. These experiments as well as the extrapolation to the island divertor are based on an extensive numerical study of plasma edge transport and radiation cooling by intrinsic and seeded impurities carried out with the fully $3 \mathrm{D}$ fluid plasma edge and kinetic neutral transport code EMC3-EIRENE [17]. These simulations predict that $\mathrm{N}_{2}$ seeding enables to actively cool the plasma edge with small penetration in the confined plasma, while $\mathrm{Ne}$ results in a deeper expansion into the confinement region of the radiation. Also, the modelling results predict a localization of the seeded impurities aligned with the magnetic structure. These predictions represent a valuable guideline for the experiments.

\section{Description of the experiment}

The Wendelstein 7-X stellarator $(W 7-X)$

$\mathrm{W} 7-\mathrm{X}$ is the largest fully optimized, super-conducting stellarator in the world with a minor radius of $0.5 \mathrm{~m}$, major radius of $5.5 \mathrm{~m}$ and magnetic field strength on axis $B=2.5 \mathrm{~T}$. W7-X is a HELIcal Advanced Stellarator (HELIAS) with strongly varying plasma cross-section, five field periods and low shear. The device consists of five nominally identical modules. Each of these is made of two flip-symmetric parts, so that in fact the device is composed of ten almost identical half-modules [20]. The poloidal symmetry plane changes within a half-module from a beanshaped cross-section at $\phi_{\mathrm{tor}}=0^{\circ}$ through a tear-shape cross-section at $\phi_{\mathrm{tor}}=12.3^{\circ}$ to a triangular cross-section at $\phi_{\mathrm{tor}}=36^{\circ}[21]$.

For the start-up campaign five poloidal graphite limiters were installed on the torus inboard side, which defined the plasma boundary and avoided significant power loads to the metallic in-vessel components [3]. For the later operation phases, the heat fluxes will be distributed over a much larger area provided by the divertor target plates in the island divertor, where pumping will also be implemented [22].

The localization of the limiters within the helicity of the plasma equilibrium and the local magnetic shear at the plasma boundary establishes a 3D boundary consisting of three distinct types of magnetic flux tubes with different target-to-target connection lengths $\left(L_{\mathrm{C}}\right)(36,43,79 \mathrm{~m})$ [17].

Two different magnetic configurations were used during the seeding discharges: the standard OP1.1 configuration and a configuration at increased rotational transform, measured by the iota $\left(i=2 \pi \cdot d \psi_{\theta} / d \psi_{\varphi}\right)$. In the standard OP1.1 configuration the 5/6 island chain is slightly inside the LCFS and the 5/5 islands, which later will establish the island divertor, are far outside of the LCFS such that no large islands are present close to the limiter. Hence, no direct heat and particle loads connected to the interaction of the islands are expected [5]. In the increased iota OP1.1 configuration the 5/5 islands move inwards and become visible in the far SOL. The 5/6 island, located inside of the last closed flux surface, is moved inwards too and is now located in the particle source region, thus 
interacting with the recycling flux as main plasma particle source [23]. The plasma heating is provided in this initial phase only by electron cyclotron resonance heating (ECRH) with 6 gyrotrons, delivering a maximum power of 4.3 MW. For the experiments described in this work the input power was set to 2-3 MW in X-mode on-axis heating providing central power deposition [24]. Central plasma parameters were $T_{\mathrm{e}} \sim 5 \mathrm{keV}, n_{\mathrm{e}} \sim 1.5 \times 10^{19} \mathrm{~m}^{-3}$.

\section{Injection system and edge diagnostics}

Figure 1 shows the position of the diagnostics used with respect to the magnetic flux tube structure. The pentagon shape of the torus is clearly visible. Machine module 1 is the upper right one, then the others follow anti-clockwise. In the bean-shaped cross-section of each module, on the inboard side, a limiter is installed. The impurities are seeded by gas injection from one of the two gas boxes installed in the device [6]. The gas boxes are located in two symmetric positions, one at the bottom of the tear-shape cross-section in module 3 and one at the top of the same cross-section in module 5. Figure $2(a)$ and $(b)$ show the position of the valve in the tear-shape cross-section in module 5 with target-to-target connection length $\left(L_{\mathrm{C}}\right)$ domains for the two magnetic configurations used in these experiments. The tear-shape cross-section in module 3 is reversed with respect to Figure $2(a)$ and $(b)$. Nitrogen is injected from the gas box in module 3, while neon from the one in module 5. The two locations are connected by the same flux tube and they lie in an upstream position with respect to the limiter target (see Figure 1). The gas is injected through a fast piezo valve (opening time of few $\mathrm{ms}$ ), which enables fast duty cycles for the gas injection and a direct control of the amount of gas utilized [25].

In the experiments under consideration here, the impurity seeding is provided by one single source. The same arrangement will be also used during the first divertor campaigns (OP1.2). In the later divertor phase (OP2) 10 toroidally symmetric gas boxes are envisaged, two in each module in the upper and lower divertor plate. Their aim is to provide a uniform cooling of the entire SOL and uniform fueling capabilities. In the experiments described in this paper, a first exploration of the seeding capabilities in the 3D plasma edge of $\mathrm{W} 7-\mathrm{X}$ is presented.

Three edge diagnostics were employed as main diagnostics to measure the variation of $T_{\mathrm{e}}$ in different locations within the SOL: the thermal He-beam and the Langmuir probe on the multi-purpose manipulator, located in two different upstream positions, and the Langmuir probes on two limiter tiles, at the downstream target position.

The He-beam measures radial profiles of $T_{\mathrm{e}}$ and $n_{\mathrm{e}}$ through line-ratio spectroscopy on thermal He [26]. The gas is injected through the gas box in module 5 . This gas box is equipped with a thin capillary nozzle for small divergence beam. The He-beam diagnostic was originally designed to measure $T_{\mathrm{e}}$ and $n_{\mathrm{e}}$ in the SOL along 8 lines-of-sight $(\mathrm{LoS})$ perpendicular to the beam propagation, with $1 \mathrm{~cm}$ spatial resolution in the radial direction [19]. However, due to unfavorable coating of the observation window originating from the plasma operation, only spatially averaged temperatures and densities were obtained. Time resolution is $25 \mathrm{~ms}$.

The multi-purpose manipulator is installed at the outboard side of the tear-shape cross-section in module 4 as shown in Figure 2. In this initial phase the manipulator was equipped with the so-called combined probe, which includes five Langmuir probe pins [27]. With the manipulator in a fixed position within the SOL, time traces of temperature and density are measured. In the experiments presented here the manipulator was parked $\sim 7 \mathrm{~cm}$ outside the LCFS providing temperature and density measurements with a time resolution of $0.2 \mathrm{~ms}$.

The limiter Langmuir probes comprise two arrays with 20 probes each installed in two tiles of the limiter in module 5 (one upper and one lower with respect to the mid plane). The probes provide radial profiles of $T_{\mathrm{e}}$ and $n_{\mathrm{e}}$ from the LCFS to $5 \mathrm{~cm}$ in the SOL with a $2 \mathrm{~ms}$ time resolution [6].

In Figure 1 are shown the magnetic field lines in the SOL for the flux tube which is fuelled by the $\mathrm{N}_{2} / \mathrm{Ne}$ injection. Such a tube connects the $\mathrm{N}_{2}$ gas puff location with the He/Ne gas puff location and the upstream Langmuir probe. Hence the He-beam and the upstream Langmuir probe lie on a flux tube that is directly fuelled by the $\mathrm{N}_{2}$ injection, whereas the limiter equipped with the Langmuir probes intercepts a flux tube which is not directly in the injection cloud of the gas inlet. This is true for both magnetic configurations used during these impurity seeded experiments. Figure 2(a) and $(c)$ show that in the standard OP1.1 magnetic configuration the valve fuels the long $L_{\mathrm{C}}$ flux tube $(79 \mathrm{~m})$ and the very edge $\left(L_{\mathrm{C}}>79 \mathrm{~m}\right)$, and both the He-beam LoS and the upstream Langmuir probe are located in the long $L_{C}$ flux tube. In the increased iota OP1.1 configuration (Figure 2(b) and $(d)$ ) the valve fuels the 5/5 island in the far SOL and the long $L_{\mathrm{C}}$ flux tube. In this configuration the He-beam LoS are predominantly in the long $L_{\mathrm{C}}$ flux tube while the upstream Langmuir probe sits inside the island. During $\mathrm{N}_{2}$ injections the He-beam and upstream Langmuir probe thus provide measurements of local cooling effects in the 3D helical SOL topology of the W7-X limiter plasmas. The cooling observed on the limiter tiles is instead due to cross-field transport between 
different flux tubes and its measurements are an indicator of global cooling effects in the SOL. During Ne experiments, since neon is injected through the same gas box as the He-beam, this diagnostic is not available. Moreover the upstream Langmuir probe was not operative so only the downstream measurements from the limiter Langmuir probes are available.

Additionally, the surface temperature of the limiter 5 is monitored by an infrared camera. The camera is a microbolometric camera (observable wavelength range 8-14 $\mu \mathrm{m}$, spatial resolution $\sim 5 \mathrm{~mm}$, frame rate $50 \mathrm{~Hz}$ ). From the surface temperature data the heat flux density is evaluated by solving a one-dimensional heat diffusion equation for bulk graphite material [28].

A two-camera bolometer system, comprising a horizontal one with 32 channels and a vertical one with 40 channels with lines-of-sight covering the entire triangular cross section $\left(\phi_{\text {tor }}=108^{\circ}\right)$, allows to measure the plasma radiation horizontally and vertically [29]. The tomographic inversion of the bolometer integrated signals is also performed leading to a 2D poloidal distribution of the plasma emissivity.

VUV/XUV spectroscopy $(\lambda=19-160 \mathrm{~nm})$ is provided by the High Efficiency XUV Overview Spectrometer (HEXOS, spectral resolution $0.026-0.18 \mathrm{~nm}$, time resolution $1 \mathrm{~ms}$ ) along a central line-of-sight at the triangular plane between modules 1 and $5\left(\phi_{\text {tor }}=324^{\circ}\right)$ looking at the inner metallic wall [30]. Visible spectroscopy $(\lambda=200$ $800 \mathrm{~nm}$ ) is provided by the $500 \mathrm{M}$ Overview Spectrometer (Czerny-Turner, $f_{\mathrm{L}} 500 \mathrm{~mm}$, spectral resolution $0.2-1$ $\mathrm{nm}$, time resolution $>10 \mathrm{~ms})$ along a central line-of-sight at the tear-shape cross-section in module $4\left(\phi_{\text {tor }}=228^{\circ}\right)$ looking at the inner metallic wall.

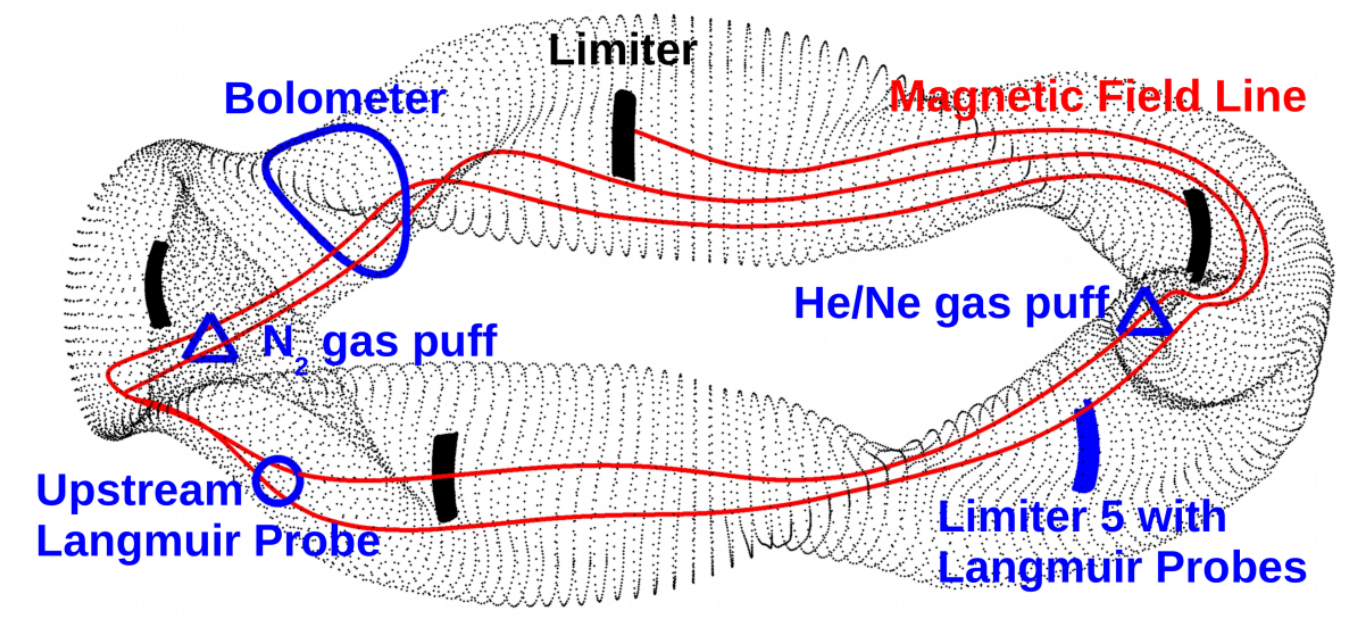

Figure 1. Field line trajectories in the scrape-off layer to show the position of the diagnostics used with respect to the magnetic flux tube structure. He-beam LoS and upstream Langmuir probe lie on flux tubes that are directly fuelled by the $\mathrm{N}_{2} / \mathrm{Ne}$ puff, while the limiter Langmuir probes are not connected to these flux bundles. 

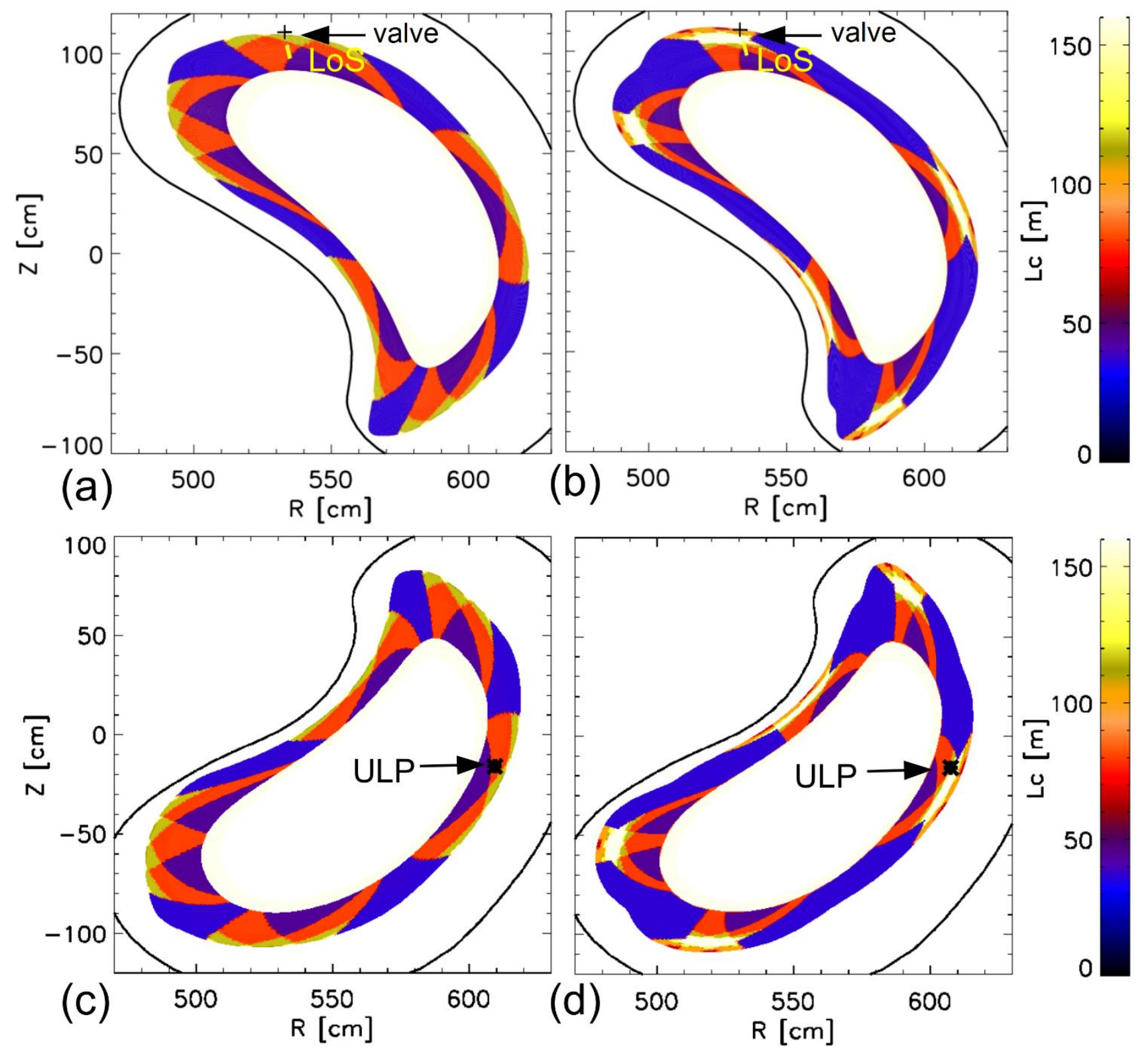

Figure 2. Cross-sections at the location of the gas box in module $5((a)$ and $(b))$ and at the location of the upstream Langmuir probe $((c)$ and $(d))$ with the target-to-target connection length domains $\left(L_{\mathrm{C}}\right)$ for the standard OP1.1 magnetic configuration $((a)$ and $(c))$ and increased iota OP1.1 configuration $((b)$ and $(d))$. The valve position is shown in $(a)$ and $(b)$ with a black cross. The radial coverage of the He-beam lines-of-sight is indicated with a yellow line in front of the valve. The gas box in module 5 is used for the He-beam diagnostic or neon injection. The gas box in module 3 (same cross-section but upside down) is used for nitrogen injection. The upstream Langmuir probe (ULP) is parked at different positions in the two configurations, shown in $(c)$ and $(d)$ with a black asterisk.

\section{Analysis of $\mathbf{N}_{2}$ seeded discharges}

$\mathrm{N}_{2}$ seeding experiments were carried out injecting gas with different particle fluxes of $2 \times 10^{19}, 4 \times 10^{19}, 6 \times 10^{19}$, $1 \times 10^{20}$ particles/s respectively. Figure 3 presents three overview plots of the main plasma parameters for a reference discharge without seeding (left), for a discharge with $\mathrm{N}_{2}$ seeding at $6 \times 10^{19} \mathrm{prt} / \mathrm{s}$ (centre) and for a discharge with $\mathrm{N}_{2}$ seeding at $1 \times 10^{20} \mathrm{prt} / \mathrm{s}$ (right). Time traces of heating power $(a)$, electron temperature $(b)$, 
electron density $(c)$, diamagnetic energy $(d)$, and total radiated power $(e)$ are shown. Effective cooling of the edge avoiding core contamination and consequent radiation collapse is found to be attainable at $6 \times 10^{19} \mathrm{prt} / \mathrm{s}$. Higher particle flux triggered a radiation collapse. An example is shown in Figure 3(right). Towards the end of the injection the radiated power level raises beyond the input power due to the high radiation from the seeded impurities which leads to a strong cooling and shrinking of the plasma and eventually to its end. ECRH power is cut off earlier than the pre-programmed time by the sniffer probes. The beginning of the end of the plasma features a pronounced neutral pressure spike, and an initially poloidally localized, rotating radiation belt before the plasma shrinks and extinguishes itself. This radiation belt is similar to the MARFE observed in tokamaks and it is described in detail in [31].

We focus now on the discharge with $\mathrm{N}_{2}$ injected at medium flow rate (Figure 3(centre)). The heating power is set at $3 \mathrm{MW}$ of ECRH. The gas injection is switched on after $100 \mathrm{~ms}$ from the start of the ECRH heating and it lasts for $200 \mathrm{~ms}$. No significant effects of the injected impurities on the core $T_{\mathrm{e}}$ were observed, which remains constant at $6 \mathrm{keV}$ for the whole discharge, whereas the temperature in the outer confined region $(r / a=0.67)$ decreases during the gas injection by $200 \mathrm{eV}$. This is a good indicator of a cooling limited to the external regions of the plasma with small impurity penetration in the inner core. Line average density increases due to wall outgassing and to the contribution of the electrons coming from the ionization of $\mathrm{N}$ and $\mathrm{N}_{2}$. Comparing the seeded discharge with the non-seeded reference discharge, the increase in $n_{\mathrm{e}}$ due to the ionization of the seeded species is of $3 \times 10^{18} \mathrm{~m}^{-3}$, in contrast the increase due to wall outgassing is of $7 \times 10^{18} \mathrm{~m}^{-3}$. Hence most of the density drive in the confined plasma is born from an uncontrolled neutral source from wall outgassing. Diamagnetic energy does not reduce due to the injection indicating no core degradation. The total radiated power increases significantly as a result of the injection, reaching $40 \%$ of the input power. 

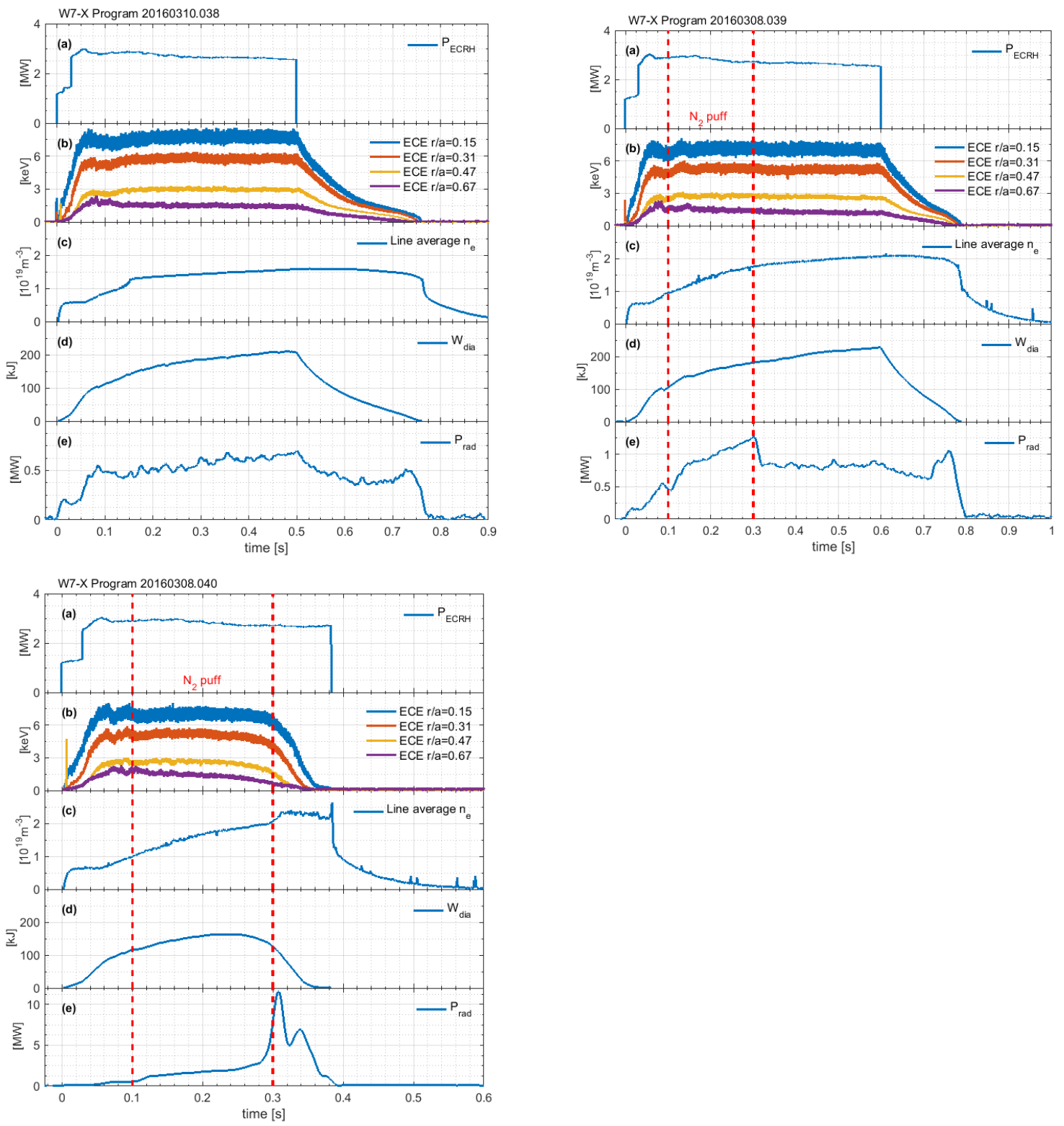

Figure 3. Overview plot of a reference discharge without injection (left), a discharge with $\mathrm{N}_{2}$ gas injection at medium flow rate $\left(6 \times 10^{19} \mathrm{prt} / \mathrm{s}\right.$, centre $)$ and a discharge with $\mathrm{N}_{2}$ gas injection at high flow rate $\left(1 \times 10^{20} \mathrm{prt} / \mathrm{s}\right.$, right $)$ which led to a radiative collapse. Time traces of: $(a)$ ECRH heating power, $(b)$ electron temperature from the Electron Cyclotron Emission diagnostic (ECE), $(c)$ line average electron density, $(d)$ diamagnetic energy, $(e)$ total radiated power. 

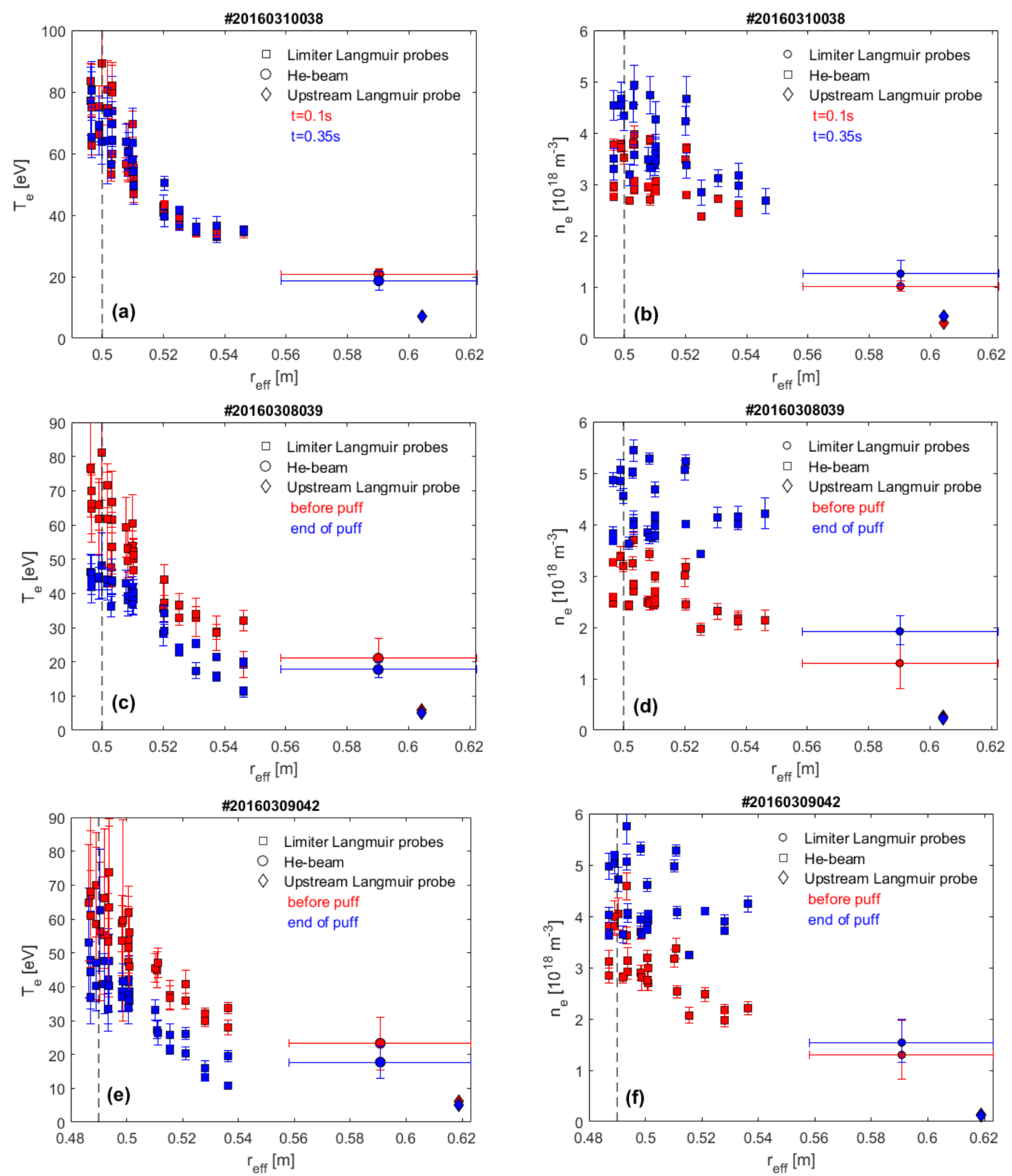

Figure 4. Radial profiles of the SOL electron temperature and density obtained combining the measurements from the three edge diagnostics (limiter Langmuir probes, He-beam and upstream Langmuir probe). (a)- $(b)$ Reference non-seeded discharge in the standard OP1.1 magnetic configuration. $(c)-(d)$ Discharge with $\mathrm{N}_{2}$ injection (constant puff 100-300 ms) in the standard OP1.1 magnetic configuration. (e)-(f) Discharge with $\mathrm{N}_{2}$ injection (pulsed puff 100-340 ms) in the increased iota OP1.1 magnetic configuration. The LCFS is also shown as a dashed black line.

Figure 4 shows the variation of the radial profiles of the electron temperature $T_{\mathrm{e}}\left(r_{\mathrm{eff}}\right)$ and density $n_{\mathrm{e}}\left(r_{\mathrm{eff}}\right)$ in the SOL following the $\mathrm{N}_{2}$ gas injection. The profiles are obtained by combining the measurements of the three edge diagnostics as a function of $r_{\text {eff. }}$ A clear reduction of $T_{\mathrm{e}}$ is seen in the whole radial length of the SOL as a consequence of the gas injection. The density increase in the SOL is driven both by wall outgassing and by ionization of the injected nitrogen. Comparing the reference non-seeded $n_{\mathrm{e}}$ profile with the seeded one, about $50 \%$ 
of the SOL density increase in the seeded discharges is due to the ionized nitrogen and $50 \%$ to wall outgassing. This is different from the line average density where the rise in the density was mainly driven by wall outgassing. Nitrogen is introduced and ionized in the SOL and there it has a stronger impact on the density rise.

The reference non-seeded discharge features a constant $T_{\mathrm{e}}$ profile (Figure $3(a)$ ) and an increasing $n_{\mathrm{e}}$ profile due to wall outgassing (Figure 3(b)). We conclude that the reduction of $T_{\mathrm{e}}$ seen in the seeded discharges is mainly given by the nitrogen radiative cooling effect with the contribution from the enhanced density rise due to the additional electrons from nitrogen ionization.

The relative $T_{\mathrm{e}}$ reduction depends on the actual placing of the diagnostic in the flux tube geometry, especially with respect to the upstream/downstream locations. He-beam and the upstream Langmuir probe both measure a decrease of the temperature of about $20 \%$. The relative cooling detected by these two edge diagnostics is the same, which is consistent with the fact that they are located in the same flux tube directly fuelled by the nitrogen gas injection. This suggests that local cooling at the upstream position is homogeneous within a single flux tube. The absolute temperature measured by the upstream Langmuir probe is lower than the He-beam average value due to the fact that the probe is located farther outside in the SOL than the He-beam. Hence, the He-beam measurement averages over higher temperatures at smaller radii.

The mapping of the Langmuir probes data on the limiter shows an exponentially decaying profile into the SOL for the temperature. Density data are more scattered because of deviations from the nominal probe area due to erosion or small dimensional changes. The error bars for both $T_{\mathrm{e}}$ and $n_{\mathrm{e}}$ result from a weighted standard deviation of the data over a $10 \mathrm{~ms}$ time window. There is a clear reduction of $T_{\mathrm{e}}$ outside of the error bars for both magnetic configuration due to the impurity injection. This reduction is seen in the region $2-5 \mathrm{~cm}$ away from the LCFS. In the region closer to the LCFS, there is a clear trend downward in the measured $T_{\mathrm{e}}$, although it stays within the increased error bars, still this is in line with an overall significant reduction of $T_{\mathrm{e}}$ due to the injection.

The relative temperature reduction at the limiter (i.e. downstream) is about $30-40 \%$, which is higher than at the upstream position of the He-beam and the upstream Langmuir probe. This means that cooling at the target, where flux tubes which are not directly fuelled by the $\mathrm{N}_{2}$ injection intersect, is observed to be stronger than upstream cooling in the fuelled flux tube. Given that the gas injection is very localized in the 3D SOL structure of W7-X with limiters, a strong localization of the radiation from injected impurities was suspected. However, the results from the temperature measurements show that the injected impurities cool the plasma effectively also in regions which are not directly connected magnetically. This is most likely due to the nature of $\mathrm{N}_{2}$ being a sticking gas species and hence, accumulation of injected gas on the limiter is likely, which results in a local and gradually increasing $\mathrm{N}_{2}$ source from the surface. Subsequent injections induce the mobilization of the nitrogen reservoir at the limiter, with consequent strong cooling in that location. This is the most likely reason for the effective and stronger cooling at the surface which is not directly connected to the injection flux tube. It was not possible to address the time dependence of this hypothesis because of the very limited experimental time (this experiment was performed in the very last week of the start-up campaign). 


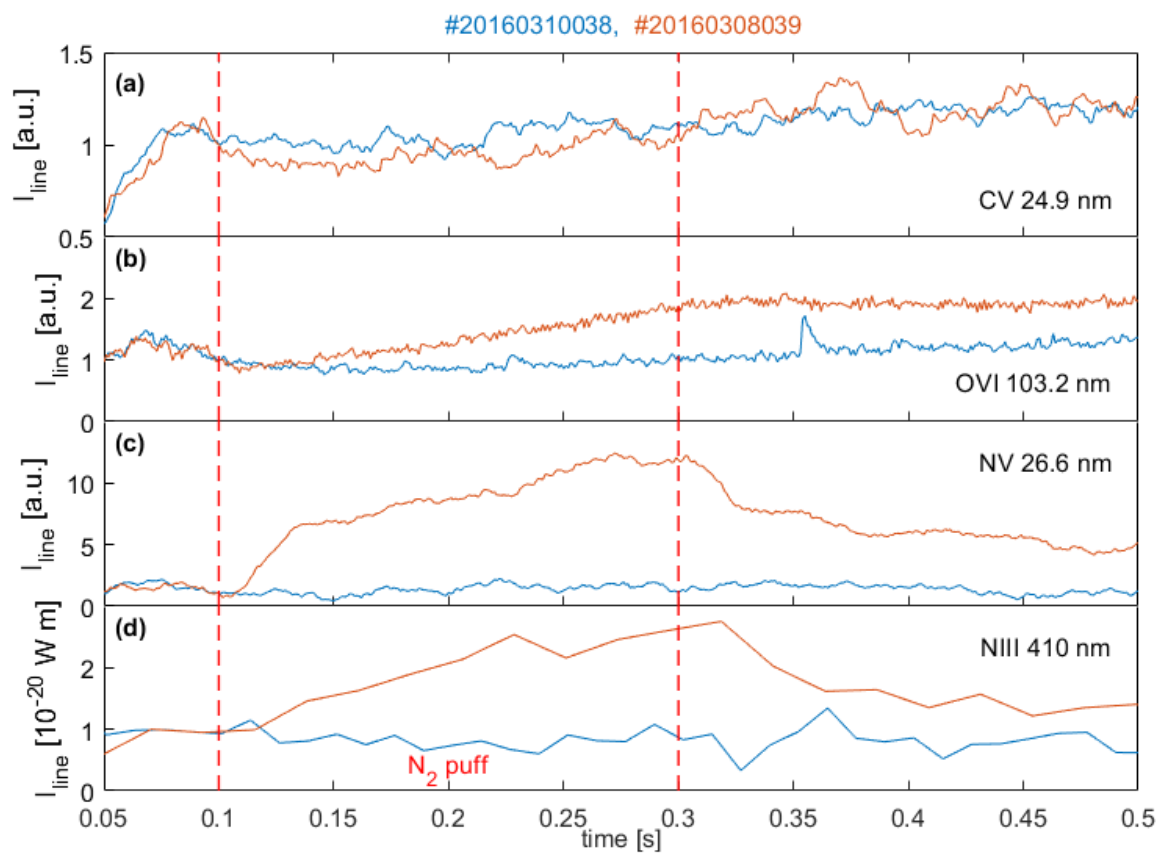

Figure 5. Line-integrated emissions in the VUV and visible range for intrinsic and extrinsic impurities measured by central line-of-sight spectrometers for a reference non-seeded discharge (blue) and a $\mathrm{N}_{2}$-seeded discharge (red). (a)-(c) HEXOS spectrometer: relative intensity. (d) $500 \mathrm{M}$ overview spectrometer: absolute intensity.

In Figure 5 VUV and visible spectroscopy shows the evolution of intrinsic $(\mathrm{C}$ and $\mathrm{O})$ and extrinsic $(\mathrm{N})$ impurity line emissions during a nitrogen seeded discharge. These lines originate mostly in the SOL. C V radiation is very weakly reduced during the seeding. As it will be shown also in the following, $\mathrm{C}$ sputtering rate is weakly impacted by the nitrogen seeding. O VI slowly increases during the seeding and then levels off at the end of the injection. The reason is an increased release of oxygen due to the contribution of the extrinsic ions also from the metallic wall. Air leak in the gas box is excluded since nitrogen content does not increase during a neon seeded discharge (see Figure 9). Nitrogen radiation raises soon after the start of the injection for both $\mathrm{N}$ V and III ions. At the valve closure the radiation slowly decreases due to the wall pumping until the saturation is reached and a baseline level of nitrogen remains in the vessel. 

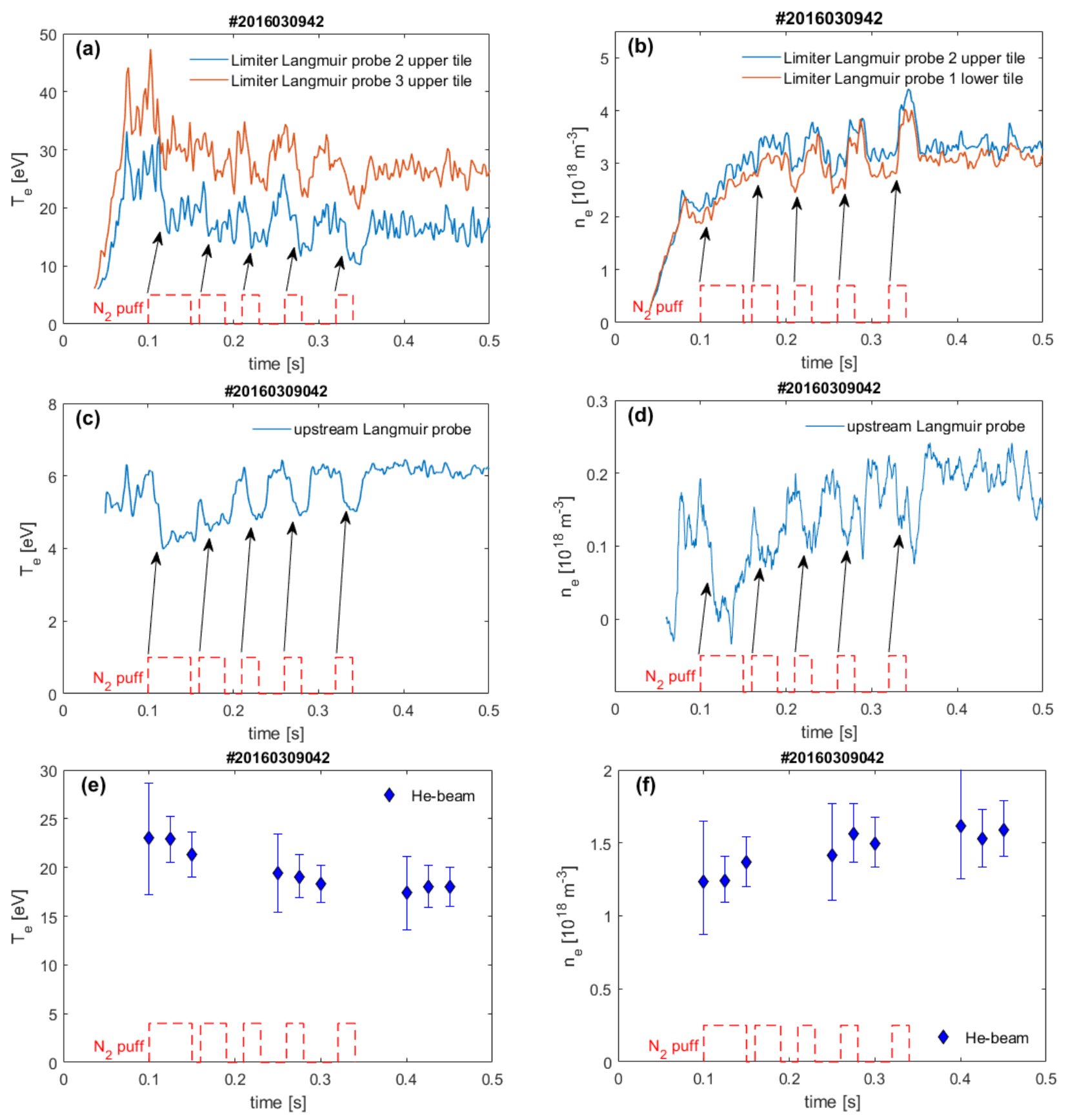

Figure 6. Pulsed $\mathrm{N}_{2}$ injection. Time evolution of the electron temperature measured by $(a)-(b)$ limiter Langmuir probes (probe 2 and 3 from the upper tile at $r_{\text {eff }}=0.536 \mathrm{~m}$ and $0.516 \mathrm{~m}$, probe 1 from the lower tile at $r_{\text {eff }}=0.528$ $\mathrm{m}),(c)-(d)$ upstream Langmuir probe, $(e)-(f)$ He-beam.

One injection experiment was conducted with a pulsed $\mathrm{N}_{2}$ gas injection to test the time response of the edge temperature. This experiment was conducted in the increased iota OP1.1 magnetic configuration. The results are presented in Figure 6, where the time traces of $T_{\mathrm{e}}$ and $n_{\mathrm{e}}$ are shown from two limiter Langmuir probes (Figure $6(a)-(b)$ ), the upstream Langmuir probe (Figure 6(c)-(d)) and the He-beam (Figure 6(e)-(f)). The $\mathrm{N}_{2}$ injection 
sequence is shown too and a clear correlation between the injection time and the temperature and density variation is visible.

The limiter Langmuir probes located far from the LCFS clearly see the modulation of the puff (the effect is visible in the 3-4 most distant probes, 2.5-4.5 cm away from the LCFS). The drop in $T_{\mathrm{e}}$ and raise in $n_{\mathrm{e}}$ are correlated with the opening/closing of the valve. The delay of $\sim 5-10 \mathrm{~ms}$ between the time of the gas injection and the $T_{\mathrm{e}} / n_{\mathrm{e}}$ response is mainly due to the valve opening time. The rapid response of the temperature variations shows that the dominant cooling action comes from the actively injected gas. The same is true for the upstream Langmuir probe which measures a variation of $T_{\mathrm{e}}$ and $n_{\mathrm{e}}$ correlated with the gas valve.

The He-beam diagnostic has a long integration time $(25 \mathrm{~ms})$ and it was not synchronized with the valve opening, so it does not allow to resolve the injection oscillations. Nevertheless, also this measurement shows a clear gradual reduction of $T_{\mathrm{e}}$ and increase in $n_{\mathrm{e}}$ indicating an accumulation of $\mathrm{N}_{2}$ in the SOL yielding the overall edge cooling effect discussed before.

Limiter Langmuir probes and He-beam show a decay of the average temperature throughout the gas injection, which does not recover to its pre-injection level after the end of the injection. This is a combination of density rise and partially recycling nitrogen which is sputtered back from the carbon wall into the SOL and transferred to different areas of the wall which had not been saturated with $\mathrm{N}_{2}$. This sustains some baseline level of nitrogen in the SOL after the injection which is confirmed by VUV and visible spectroscopy shown earlier.

The upstream Langmuir probe measures a full recovery of $T_{\mathrm{e}}$ immediately after the gas injection. In the increased iota scenario the probe mounted on the mid-plane manipulator was parked in a far outwards position (see Figure $2(d)$ ) where the $5 / 5$ island is present. This magnetic island receives less impurities through limiter surface erosion compared to the SOL flux tubes, which are connected to the limiters and facing the confinement. The upstream Langmuir probe measures a diminishing density in correspondence with the incoming nitrogen flow. In the far SOL nitrogen is weakly ionized and hydrogen recombination is enhanced by the temperature reduction due to the injection, thus reducing the electron density measured by the probe.

\section{Analysis of Ne seeded discharges}

Neon impurity seeding experiments were performed at flow rates of $3.5 \times 10^{19}, 7 \times 10^{19}$ and $9 \times 10^{19}$ particles/s in the standard OP1.1 magnetic configuration. Figure 7 presents three overview plots of the main plasma parameters for a reference discharge without seeding (left), for a discharge with Ne seeding at $3.5 \times 10^{19} \mathrm{prt} / \mathrm{s}$ (centre) and for a discharge with $\mathrm{Ne}$ seeding at $7 \times 10^{19} \mathrm{prt} / \mathrm{s}$ (right). Time traces of heating power $(a)$, electron temperature $(b)$, electron density $(c)$, diamagnetic energy $(d)$ and total radiated power $(e)$ are shown. When injected at high flow rates $\left(7 \times 10^{19}\right.$ and $\left.9 \times 10^{19} \mathrm{prt} / \mathrm{s}\right)$ neon triggered a radiation collapse similar to the one already described for nitrogen (see Figure 7(right)). Only when injected at low flow rate $\left(3.5 \times 10^{19} \mathrm{prt} / \mathrm{s}\right)$ neon did not lead to a collapse.

We focus now on the discharge with Ne injected at low flow rate (Figure 7(centre)). The heating power is set at 2 MW of ECRH. The gas injection is switched on after $200 \mathrm{~ms}$ from the start of the ECRH heating and it lasts for $300 \mathrm{~ms}$. Electron temperature in the core decreases by $\sim 10 \%$ during the gas injection, whereas the external channel of the ECE system $(r / a=0.67)$ reveals a drop of $40 \%$ of $T_{\mathrm{e}}$ caused by the injected neon. $T_{\mathrm{e}}$ keeps decreasing also after the end of the injection in the whole plasma volume thus meaning a strong impact of neon as radiating away the plasma energy. The line average density increases almost entirely due to wall outgassing. Comparing the seeded discharge with the non-seeded reference discharge, the increase in $n_{\mathrm{e}}$ due to the ionization of the seeded species is of $5 \times 10^{17} \mathrm{~m}^{-3}$, in contrast the increase due to wall outgassing is of $8 \times 10^{18} \mathrm{~m}^{-3}$. Hence we conclude that the density drive in the confined plasma is born from an uncontrolled neutral source from wall outgassing. Diamagnetic energy is reduced by $50 \%$ at $t=6 \mathrm{~s}$ with respect to the non-seeded discharge as an indicator of core degradation. Total radiated power increases significantly due to the impurities injection, it keeps raising after the closure of the valve reaching $70 \%$ of the input power at $0.58 \mathrm{~s}$.

Figure 8 shows the response of the SOL $T_{\mathrm{e}}$ and $n_{\mathrm{e}}$ to the neon injection as measured by the limiter Langmuir probes, along with a reference non-seeded discharge. A significant reduction of $20-30 \mathrm{eV}$ corresponding to a $50 \%$ relative reduction is measured by the probes. At the same time the density in the SOL increases by $80 \%$ due to the ionization of neon since the reference discharge features a constant density profile. We conclude that the reduction of $T_{\mathrm{e}}$ seen in the neon seeded discharge is given by a combination of the density rise and of the neon radiative cooling effect. The temperature continues to lower after the end of the gas injection (see Figure 8(e)) as measured 
by the ECE for the confined plasma. This suggests strong recycling of Ne from the limiter surface with consequent radiation and cooling of the plasma after the closure of the valve.

In Figure 9 VUV and visible spectroscopy shows the evolution of intrinsic (C and $\mathrm{O})$ and extrinsic $(\mathrm{Ne})$ impurity line emissions during a neon seeded discharge. These lines originate in the edge plasma close to the LCFS and in the SOL. C and O line radiation behaves in a way similar to the nitrogen seeding case. $\mathrm{C} \mathrm{V}$ is not affected by the neon injection. O VI raises during the injection due to increased sputtering by neon also from the metallic wall. A constant N III radiation level proves that the increased O content does not come from an air leak in the gas box. Neon radiation raises soon after the start of the injection for both N VIII and II ions. At the valve closure the radiation levels off without diminishing due to recycling of $\mathrm{Ne}$ from the wall surfaces.

Neon is seen to affect consistently the entire plasma domain, even when injected in small amount, radiating away most of the input power and cooling the plasma from the SOL to the central region. After the end of the gas injection neon continues to radiate, suggesting high recycling. This is consistent with what has been previously observed in LHD where a strong core radiation was measured after stopping the Ne injection. The reason found was the high recycling of neon which gradually enhanced the concentration in the core region [16].

Conversely, in the limiter tokamak TEXTOR-94 a high confinement regime characterized by high $\beta$ and high density was achieved by strong edge radiation cooling with neon seeding [13]. No deleterious effects of the neon seeding on fusion reactivity and plasma stability were observed. The indispensable tool to obtain a stable radiating layer with neon seeding was the feedback control of the neon inlet valve together with the toroidal pumped limiter (ALT-II) of TEXTOR which is acting as the sink for the neon in the feedback loop. Ne seeding was feedback controlled in order to maintain the radiated power fraction $f_{\text {rad }}=P_{\text {rad }} / P_{\text {in }} \sim 80 \%$.

Neither of these two prerequisites were available at Wendelstein $7-\mathrm{X}$ in order to obtain a stable radiative edge with neon seeding. The gas valve is strongly localized, which has been already demonstrated to be detrimental for confinement [32]. The amount of seeded gas is not feedback controlled, resulting in too much neon being injected with consequent degradation of the discharge. Neon is not properly exhausted by the turbo-molecular pumps installed at W7-X, thus it accumulates in the vessel after the closure of the valve. For the future divertor campaigns, more gas boxes symmetrically distributed around the torus are envisaged, along with a feedback-controlled gas injection system: this will help to reach a stable radiative mantle using neon. 

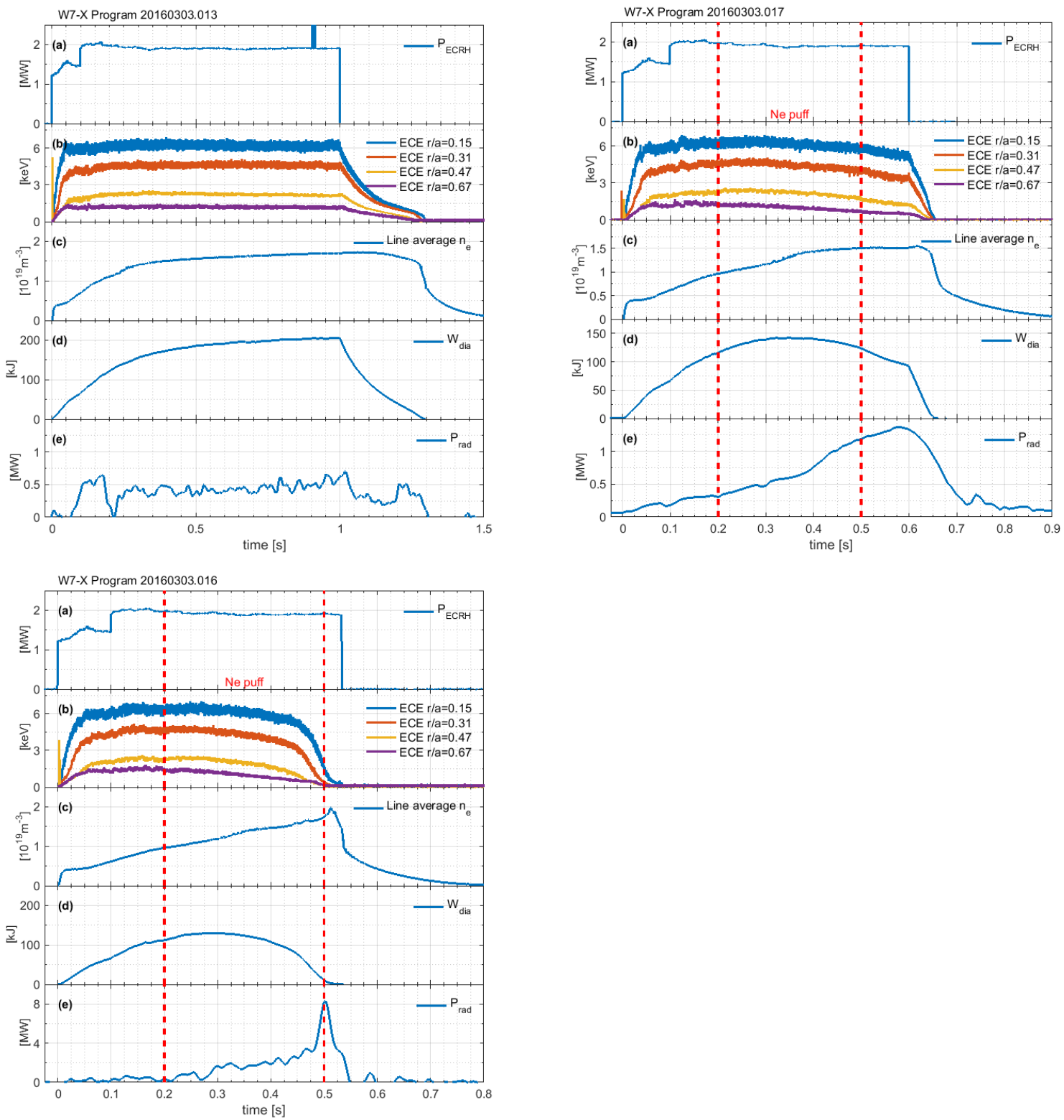

Figure 7. Overview plot of a reference discharge without injection (left), a discharge with $\mathrm{Ne}$ gas injection at low flow rate $\left(3.5 \times 10^{19} \mathrm{prt} / \mathrm{s}\right.$, centre $)$ and a discharge with Ne gas injection at high flow rate $\left(7 \times 10^{19} \mathrm{prt} / \mathrm{s}\right.$, right $)$ which led to a radiative collapse. From top to bottom: $(a)$ ECRH heating power, $(b)$ electron temperature from ECE, $(c)$ line average electron density, $(d)$ diamagnetic energy, $(e)$ total radiated power. 

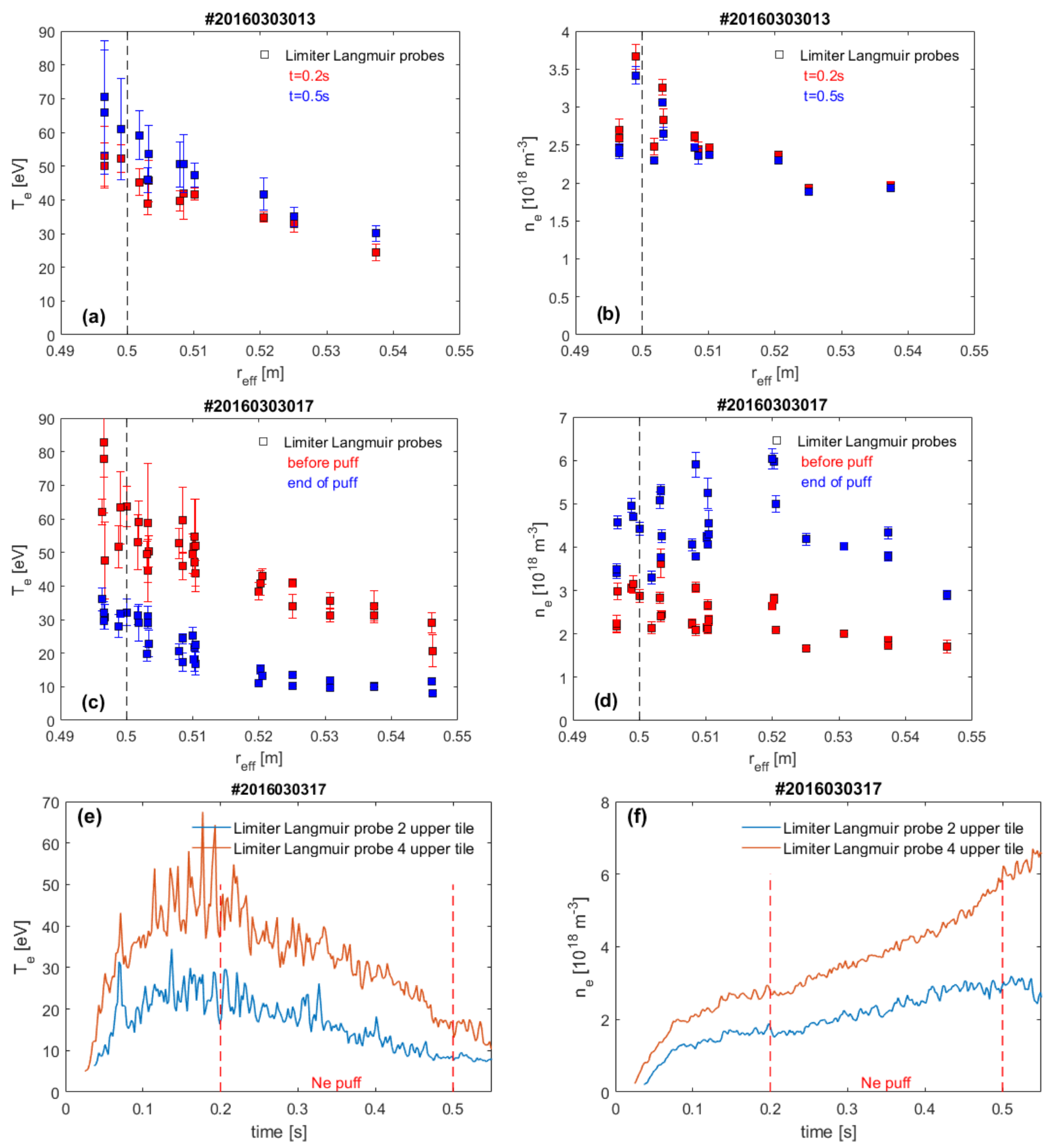

Figure 8. Electron temperature and density measured by the limiter Langmuir probes. $(a)-(b)$ radial profiles for a reference non-seeded discharge. $(c)-(d)$ radial profiles before (red) and at the end (blue) of the Ne injection (LCFS is shown as a dashed black line). (e)-(f) time traces of two probes from the upper tile array (probe 2 at $r_{\text {eff }}=0.546$ $\mathrm{m}$ and probe 4 at $r_{\text {eff }}=0.52 \mathrm{~m}$ ). 


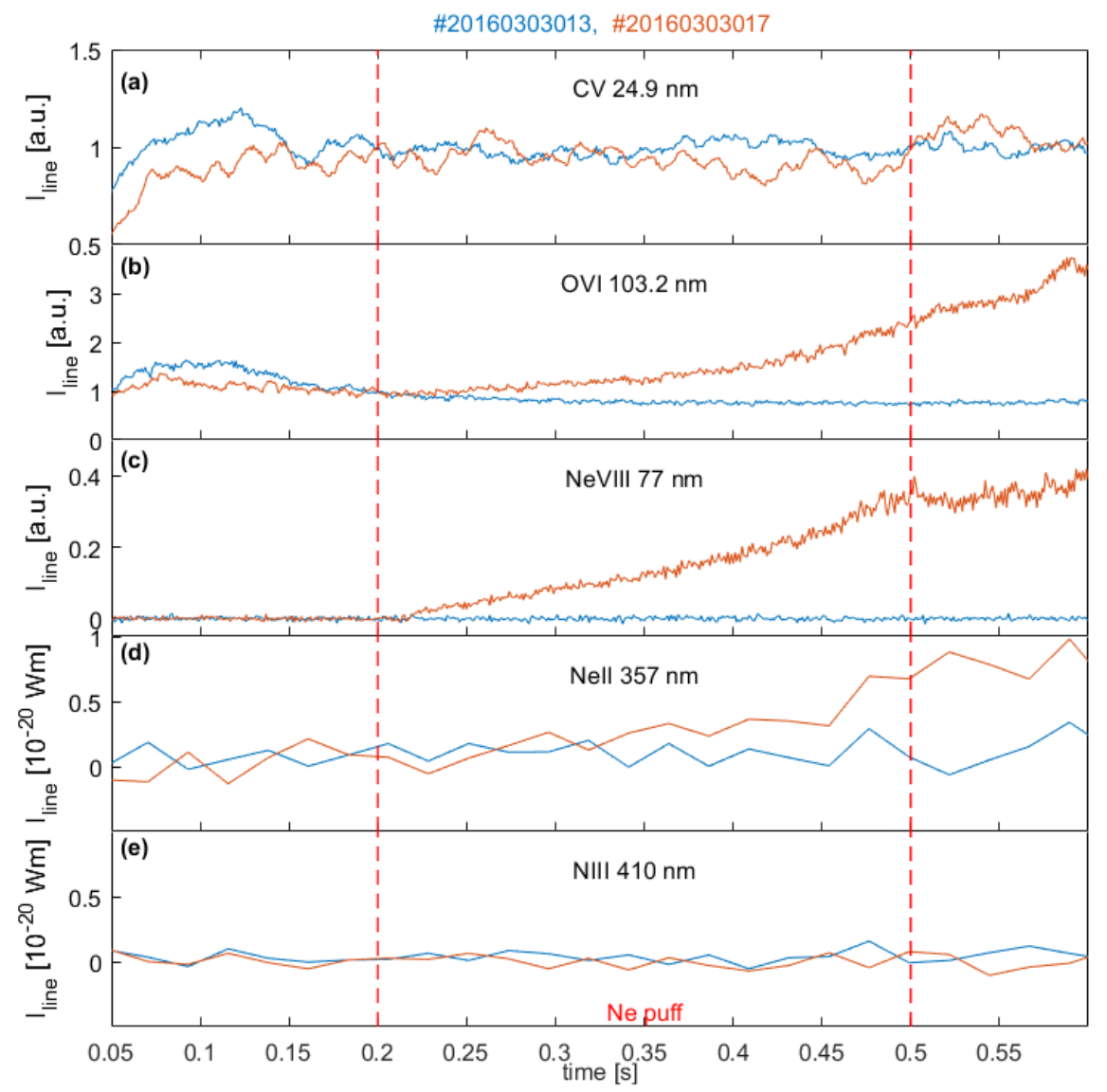

Figure 9. Line-integrated emissions in the VUV and visible range for intrinsic and extrinsic impurities measured by central line-of-sight spectrometers for a reference non-seeded discharge (blue) and a Ne-seeded discharge (red). (a)-(c) HEXOS spectrometer: relative intensity. (d-e) $500 \mathrm{M}$ overview spectrometer: absolute intensity.

\section{Analysis of radiation distribution}

Nitrogen injection

The radiated power is calculated from LoS-integrated measurements of the horizontal bolometer camera [33]. In Figure 10 the time trace of the total radiated power during a $\mathrm{N}_{2}$ injection is shown. During non-seeded stationary plasma phases the radiative power loss fraction $P_{\text {rad }} / P_{\text {in }}$ is around $25 \%$. With the introduction of nitrogen this fraction raises up to $45 \%$ during the active injection. After the end of the injection the radiative power loss fraction lowers to a level of $30 \%$ due to the remaining nitrogen in the device.

2D emissivity distributions have been obtained using a Bayesian tomographic method based on the bolometer measurements. This method uses Gaussian processes to model the emission distribution and then uses Bayesian inference to calculate a posterior Gaussian process describing the range of possible tomographic reconstructions given the data and model assumptions [34]. The tomographic inversions have been done in the Minerva framework [35].

Figure 11 shows the 2D emissivity distributions for the $\mathrm{N}_{2}$ injection discharge at different times. In Figure 12 the radial profiles of the radiated emissivity are also shown. Before the injection the radiation comes from a broad region located inside the confined region with the peak value at $r / a=0.8$. This radiation is mainly emitted by low- 
$\mathrm{Z}$ impurities such as $\mathrm{C}$ and $\mathrm{O}$ [33]. During the $\mathrm{N}_{2}$ injection the radiation peak moves outwards at the position of the LCFS $(r / a=1)$. Nitrogen seems to radiate more outwards with respect to intrinsic impurities. Moreover the radiating layer becomes more strongly localized in the lower part of the cross-section. The bolometer array is very close to the injection point in module 3 and the flux tube which is directly fuelled by nitrogen lies at the bottom of the cross-section measured by the bolometer lines-of-sight (see Figure 1). This could explain why during the gas injection the radiation is concentrated in a region at the bottom of the poloidal section. After the injection is stopped the plasma reaches an equilibrium in which the walls get saturated by nitrogen. The radiation in this phase is emitted by a combination of intrinsic impurities and remaining nitrogen and the corresponding radial emissivity profile becomes broader, similarly to the pre-injection phase, though with a higher peak value.

\section{Neon injection}

2D emissivity distributions and corresponding radial profiles have been computed also for the neon seeded discharge presented before and are depicted in Figure 14 and Figure 15. Before the gas injection, the radiation layer is broadly distributed in the outer confined region with a peak value at $r / a=0.8$ as previously discussed. During the neon injection, the radiation zone shrinks and peaks at $r / a=0.9$. After the end of the gas injection the radiation belt is still present in the same position with similar high intensity as during the gas injection. This result suggests that neon tends to accumulate in the plasma and it recycles on the wall surfaces without any significant exhaust from the system, keeping the level of radiation high after the end of the gas injection. This is also a reasonable assumption given that the exhaust efficiency of the turbo-molecular pumps for $\mathrm{Ne}$ is small. Hence, the injected gas is not exhausted and is continuously available for repetitive cooling after recycling. $\mathrm{N}_{2}$ in contrast has a lower recycling coefficient due to its sticking and hence is effectively exhausted on the time scales of the discharge by attachment the wall surfaces and hence it is not available as much for repetitive cooling.

The observation that the radiation belt is more poloidally symmetric than in the nitrogen case can be addressed through several hypothesis. The first is that neon is injected into the chamber from the gas box which is located $168^{\circ}$ toroidally away from the bolometer array: neon impurities travel a longer distance than nitrogen before reaching the bolometer and hence they can redistribute in the neighbouring flux tubes. The second is the limited numbers of diagnostic channels available for the tomographic reconstruction for this discharge. This necessitates a stronger constrain of symmetry within a flux surface imposed in the tomographic algorithm. Last but not least, the process of recycling and repetitive cooling of neon described before yields a stronger global distribution of the $\mathrm{Ne}$ which further reduces the localization effect.

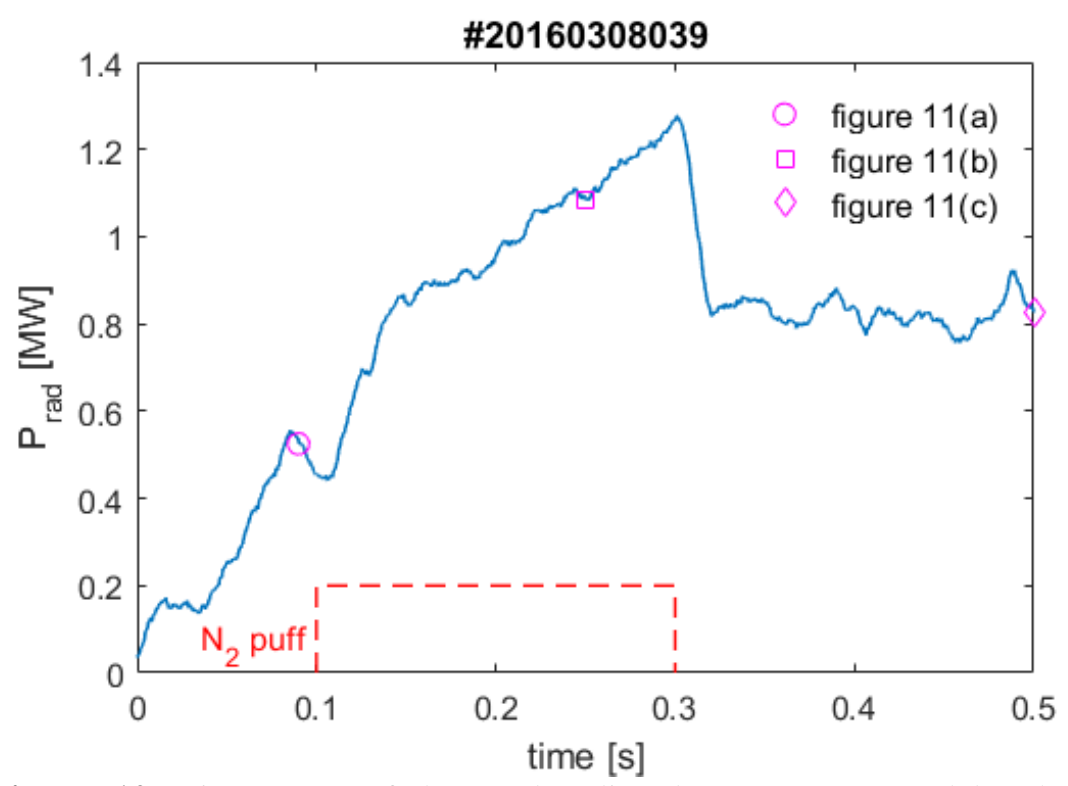

Figure 10. Time trace of the total radiated power measured by the bolometer for a $\mathrm{N}_{2}$ injection. Estimated uncertainty is $\pm 10 \%$. Magenta markers refer to the emissivity tomographic reconstructions in Figure 11 . 

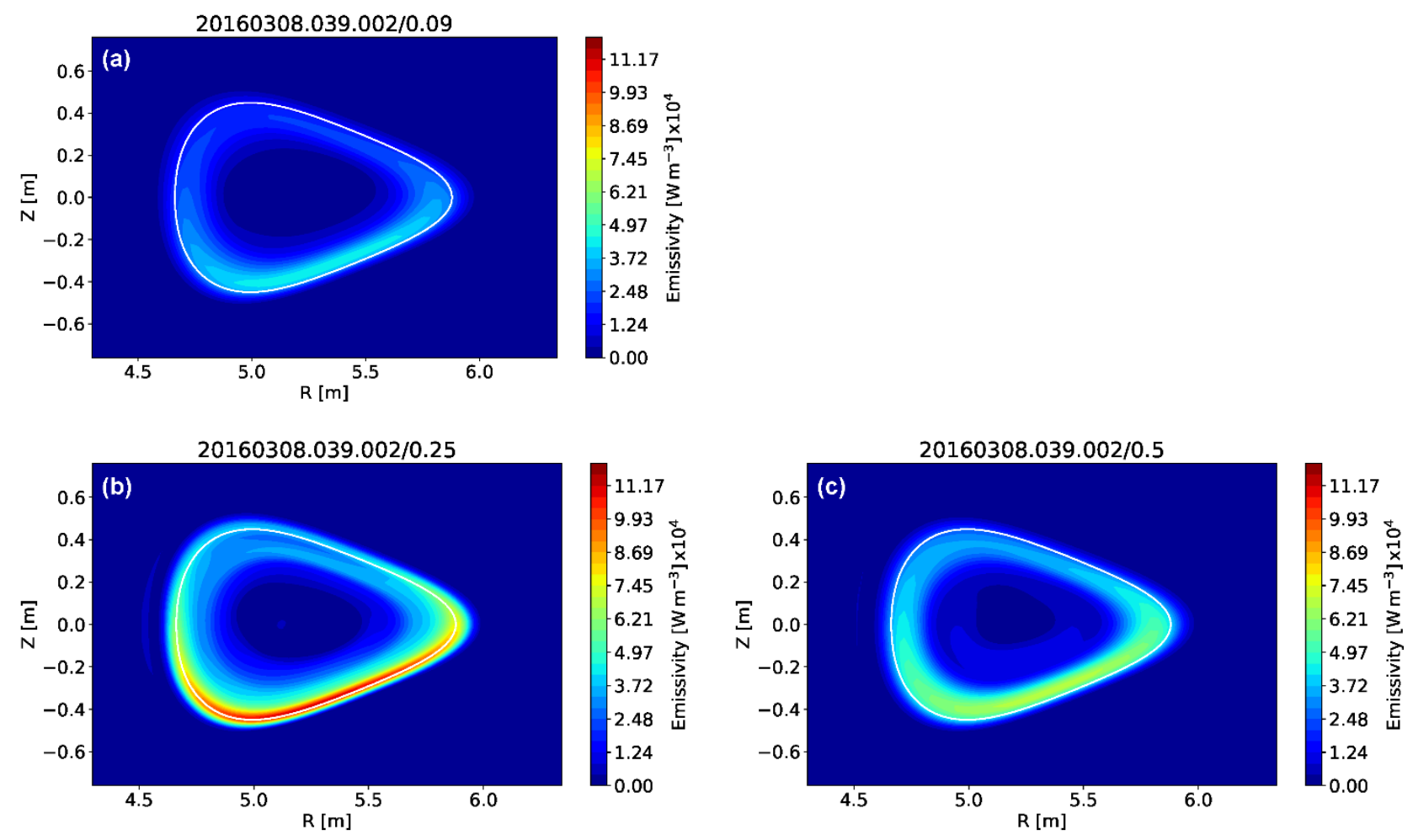

Figure 11. Tomographic reconstruction of the radiated power emissivity in a $\mathrm{N}_{2}$ seeded discharge. (a) before the injection $(0.09 \mathrm{~s}),(b)$ during the injection $(0.25 \mathrm{~s}),(c)$ after the injection $(0.5 \mathrm{~s})$. LCFS is shown in white.

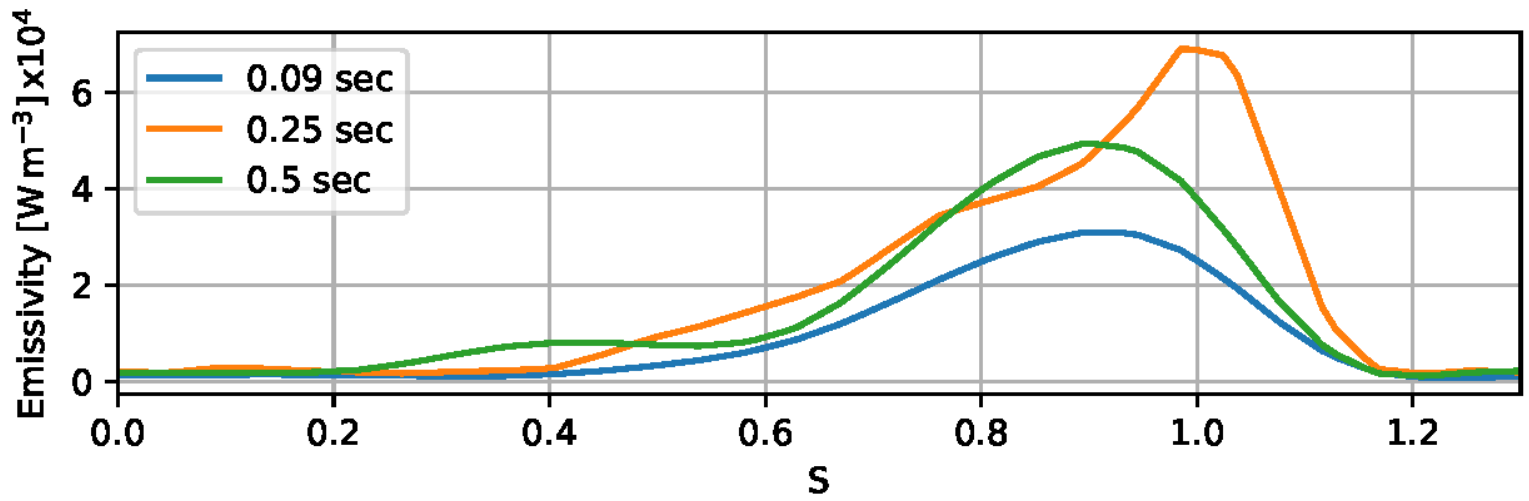

Figure 12. Radial profile of the radiated emissivity before $(0.09 \mathrm{~s})$, during $(0.25 \mathrm{~s})$ and after $(0.5 \mathrm{~s})$ a $\mathrm{N}_{2}$ seeded discharge as a function of the normalized radius $s=r / a$. 


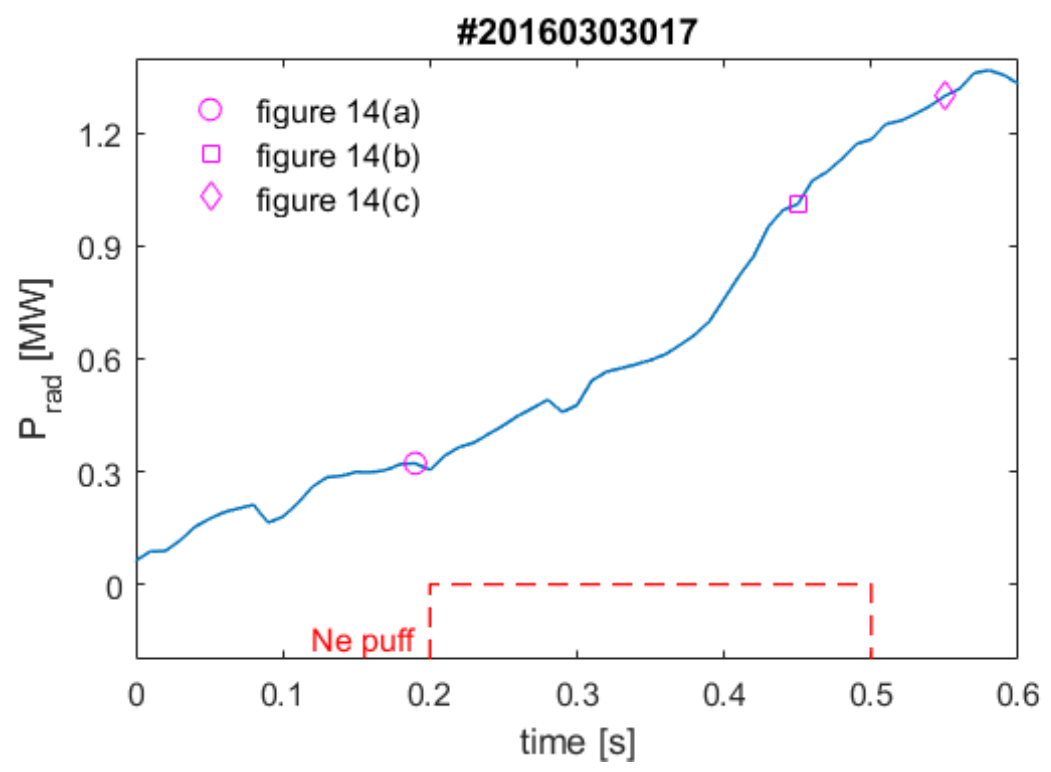

Figure 13. Time trace of the total radiated power measured by the bolometer for a Ne injection. Estimated uncertainty is $\pm 10 \%$. Magenta markers refer to the emissivity tomographic reconstructions in Figure 14 .
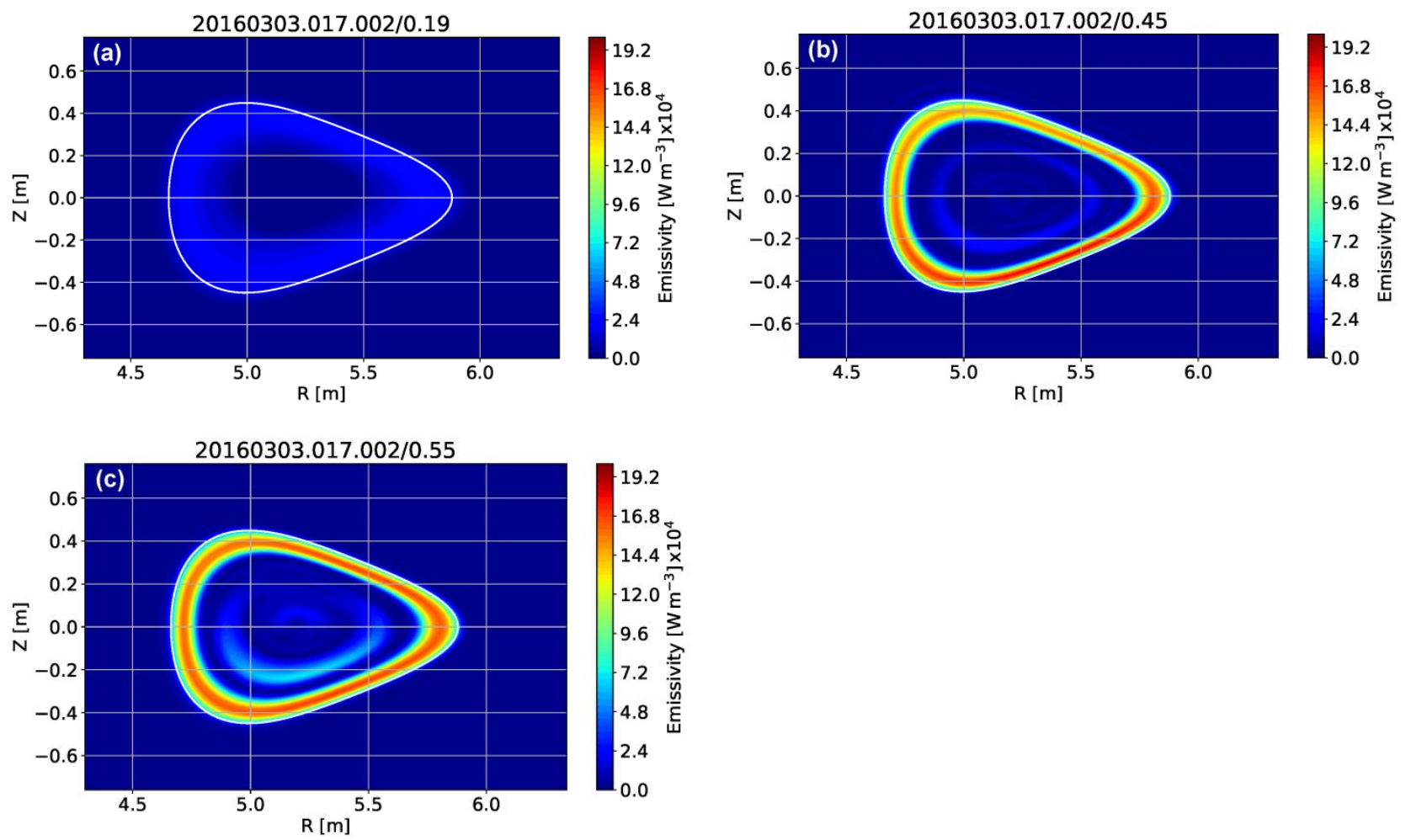

Figure 14. Tomographic reconstruction of the radiated power emissivity in a Ne seeded discharge. ( $a$ ) before the injection $(0.19 \mathrm{~s}),(b)$ during the injection $(0.45 \mathrm{~s}),(c)$ after the injection $(0.55 \mathrm{~s})$. LCFS is shown in white. 


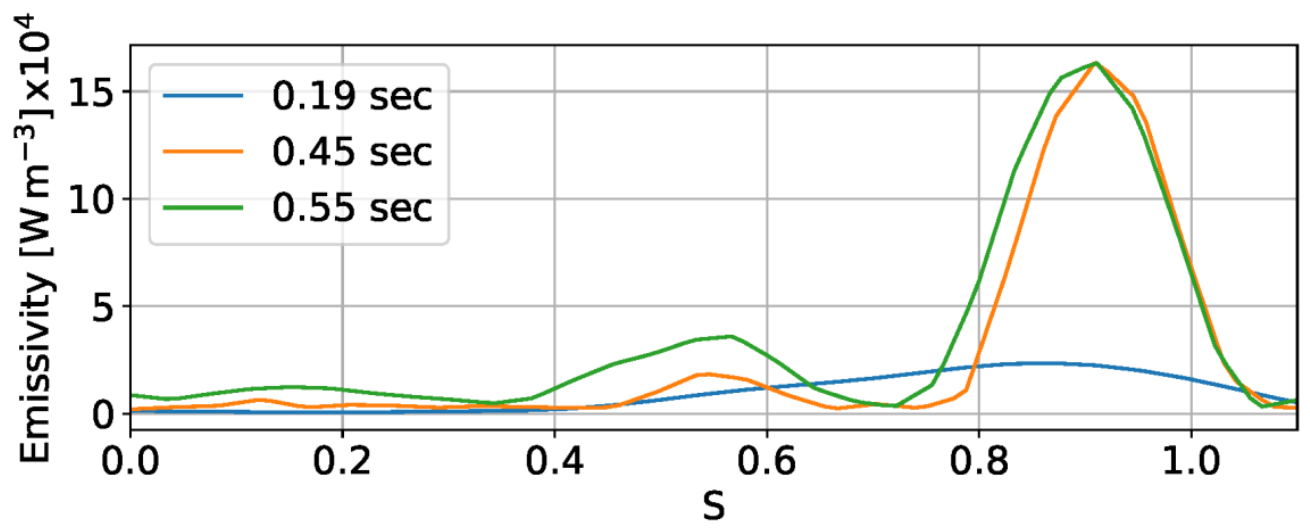

Figure 15. Radial profile of the radiated emissivity before $(0.19 \mathrm{~s})$, during $(0.45 \mathrm{~s})$ and after $(0.55 \mathrm{~s})$ a $\mathrm{Ne}$ seeded discharge as a function of the normalized radius $s=r / a$.

\section{Analysis of limiter heat fluxes}

Heat flux densities on limiter 5 are computed from measurements of the limiter surface temperature acquired with an infrared camera. Figure 16 shows the impact of the nitrogen seeding through the discharge-to-discharge evolution of the total heat load on the limiter, computed integrating the heat flux density over the limiter surface. A clear impact of the seeding is visible in the reduction of the load with increasing number of $\mathrm{N}_{2}$ seeded discharges. Consecutive $\mathrm{N}_{2}$ seeded discharges increase the global $\mathrm{N}$ concentration in the device leading to an enhanced mitigation of the heat load on the limiters. This heat load reduction lasts for the following shots with no N injection, indicating the presence of substantial remaining $\mathrm{N}$ in the device. This $\mathrm{N}$ legacy effect was already observed in other C-wall devices such as JET [36]. A possible explanation for the $\mathrm{N}$ lasting for a long time in the vessel is surface dilution of the PFC material by nitrogen leading either to N-containing layers or to nitride formation [37]. He-cleaning discharges were performed in between the experimental sequence and led to the removal of the $\mathrm{N}$ deposited on the wall and to the reestablishment of the wall conditions prior to the $\mathrm{N}_{2}$ injections. Visible spectroscopy confirms the nitrogen legacy by the prolonged appearance of a nitrogen source over several discharges without active nitrogen seeding, measured in situ by a N II line. Figure 17(a) shows the discharge evolution of C III $465 \mathrm{~nm}$ O II $459 \mathrm{~nm}$ and N II $500.5 \mathrm{~nm}$ for a series of N seeded shots followed by some shots without $\mathrm{N}$ injection. A high level of $\mathrm{N}$ is observed to last for the consecutive discharges after the $\mathrm{N}$ seeded shots until a series of He cleaning discharges is performed. At the same time the oxygen content is observed to increase following consecutive $\mathrm{N}_{2}$ seeded discharges. The seeded species seems to contribute to the release of oxygen also from the metallic wall. Carbon sputtering is instead weakly affected by nitrogen, as we observe a small variation of $\mathrm{C}$ radiation during repetitive seeded discharges. Carbon sputtering by main ions is reduced due to the lower energy of the impinging ions due to plasma cooling, at the same time this reduction is compensated by the additional, chemical and physical, sputtering by nitrogen and by the dilution of the CFC surface by N providing nitrogen-containing carbon layers [37]. Additionally, the C III emission is also altered by variations in $n_{\mathrm{e}}$. It is not possible with the present tools to determine which effect dominates over the other. We can simply say that the carbon sputtering is weakly impacted by the seeding. This is also seen during neon seeding as shown in Figure $17(b)$. Carbon line emission does not vary during repetitive neon seeded discharges. Oxygen emission is weakly increased due to the contribution of the extrinsic ions in the oxygen removal from the walls. 


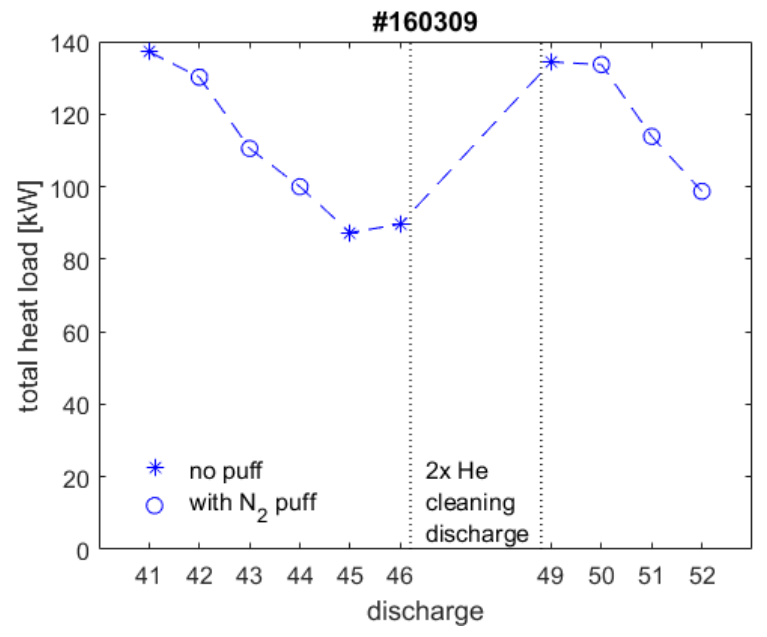

Figure 16. Discharge-to-discharge evolution of the total heat load on limiter 5, averaged over the discharge flattop.
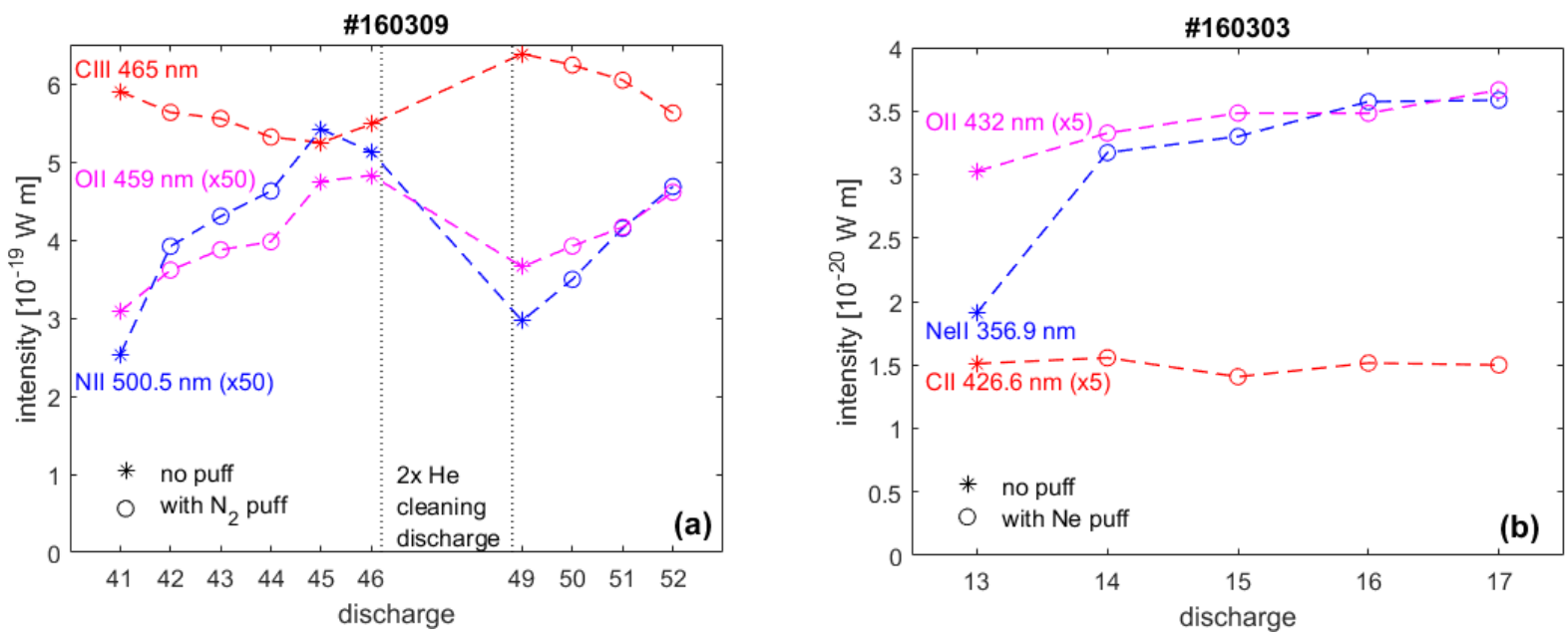

Figure 17. Discharge-to-discharge evolution of line-integrated emissions from intrinsic and extrinsic impurities during a series of $\mathrm{N}_{2}(a)$ and $\mathrm{Ne}(b)$ seeding discharges. Measurements from the $500 \mathrm{M}$ overview spectrometer averaged over the discharge flat-top.

\section{Summary and Conclusions}

An overview of observations from the first radiative edge cooling experiments on Wendelstein 7-X limiter startup phase has been presented. Injections of nitrogen and neon were carried out from two gas boxes in two toroidal positions and the effects on the edge temperature, density, radiated power and heat loads on the limiter were observed. A set of three edge temperature diagnostics located in different positions within the SOL allows to characterize the nature of the cooling, discerning local vs. global cooling. The bolometer diagnostic provides insight into the radial position of the radiation layer and to the extent possible by the spatial resolution of the system also into the poloidal structure of the radiation. An infrared camera looking at the limiter gives information about the mitigation of the heat loads on the limiter tiles.

Injection of nitrogen impurities in the edge of the plasma leads to a reduction of the electron temperature in the SOL and to a mitigation of the heat load on the limiters. The temperature decrease is not uniform in the SOL, in particular it is found that cooling at the downstream limiter position is stronger than local upstream cooling in the 
same flux tube that is fuelled by the gas valve. This is due to cross field transport into the limiter flux tubes, followed by $\mathrm{N}_{2}$ accumulation and generation of a local source at the limiter surface. Nitrogen has been shown to be a good choice for short pulse cooling: variation of temperature and radiated power are fast correlated with the opening/closing of the gas valve. Both react quickly with the gas being injected and in the same way they restore soon after the valve closing. This is very promising for the foreseen feedback-controlled gas injection being implemented for the next divertor campaigns.

Neon has been also analysed, which has different physical properties, in particular it has a larger number of radiating energy levels and a high recycling from carbon surfaces. Neon is injected with a particle influx in the ratio of $1 / 3$ with respect to atomic nitrogen, providing under comparable plasma conditions a similar cooling in the SOL but a higher radiated power. The cooling extends also in the confined region leading to a degradation of the discharge. Moreover the radiation and the consequent cooling continue after the end of the gas injection indicating strong recycling.

These experiments served as an important guideline for the next divertor campaigns. For those will be crucial to identify a proper way to execute radiative edge cooling experiments in order to both mitigate the heat loads on the plasma facing components and facilitate the transition to the detachment regime. In this regard extensive experiments are planned to be carried out in the new divertor configuration campaigns, using both $\mathrm{N}$ and $\mathrm{Ne}$ but also $\mathrm{Ar}$ (in later high-performance operation/H-mode also $\mathrm{Kr}$ or $\mathrm{Xe}$ ).

\section{Acknowledgements}

This work was supported in part by the U.S. Department of Energy (DoE) under grant DE-SC0014210 and by discretional funding of the Department of Engineering Physics and the College of Nuclear Engineering at the University of Wisconsin - Madison, USA. The publisher, by accepting the article for publication acknowledges, that the United States Government retains a non-exclusive, paid-up, irrevocable, worldwide license to publish or reproduce the published form of this manuscript, or allow others to do so, for United States Government purposes. This work has been carried out within the framework of the EUROfusion Consortium and has received funding from the Euratom research and training programme 2014-2018 and 2019-2020 under grant agreement No 633053. The views and opinions expressed herein do not necessarily reflect those of the European Commission.

\section{References}

[1] Wolf R et al 2017 Nucl. Fusion 57102020

[2] Klinger T et al 2017 Plasma Phys. Control. Fusion 59014018

[3] Bozhenkov S, Effenberg F, Feng Y, Geiger J, Hartmann D A, Hölbe H, Pedersen T S and Wolf R C 201441 st EPS Conf. on Plasma Physics P1.080

[4] König R et al 2015 J. Instrum. 10 P10002

[5] Pedersen T S et al 2015 Nucl. Fusion 55126001

[6] Krychowiak M et al 2016 Rev. Sci. Instrum. 87 11D304

[7] Geiger J et al 2013 Plasma Phys. Control. Fusion 55014006

[8] Hölbe H, Pedersen T S, Geiger J, Bozhenkov S, König R, Feng Y, Lore J and Lumsdaine A 2016 Nucl. Fusion 56026015

[9] Maddison G P et al 2011 Nucl. Fusion 51042001

[10] Wang H Q et al 2016 58th Annual Meeting of the APS Division of Plasma Physics

[11] Reinke M L, Hughes J W, Loarte A, Brunner D, Hutchinson I H, LaBombard B, Payne J and Terry J L 2011 J. Nucl. Mater. 415 S340-4

[12] Kallenbach A et al 2010 Plasma Phys. Control. Fusion 52055002

[13] Messiaen A M et al 1997 Physics of Plasmas 41690

[14] Mukai K et al 2015 Nucl. Fusion 55083016

[15] Tanaka H et al 2016 Nuclear Materials and Energy 12 241-246

[16] Morisaki T et al 2015 J. Nucl. Mater. 463 640-643

[17] Effenberg F et al 2017 Nucl. Fusion 57036021

[18] Winters V et al 2017 Phys. Scr. 2017 T170

[19] Barbui T, Krychowiak M, König R, Schmitz O, Muñoz Burgos J M, Schweer B and Terra A 2016 Rev. Sci. Instrum. 87 11E554 
[20] Bosch H-S et al 2013 Nucl. Fusion 53126001

[21] Renner H et al 2000 Nucl. Fusion 401083

[22] Renner H et al 1993 20th EPS Conf. on Plasma Physics

[23] Stephey L et al 2018 Physics of Plasmas 25062501

[24] Marsen S, Stange T, Gellert F, Höfel U, Laqua H P, Moseev D and Preynas M 2016 43rd EPS Conf. on Plasma Physics P4.002

[25] Griener M et al 2017 Rev. Sci. Instrum. 88033509

[26] Schweer B, Brix M and Lehnen M 1999 J. Nucl. Mater. 266 673-678

[27] Drews P et al 2017 Nucl. Fusion 57126020

[28] Niemann H, Jakubowski M, Pedersen T S, König R, Wurden G A, Effenberg F and Zhang D 2016 43rd EPS Conf. on Plasma Physics $\mathrm{P} 4.005$

[29] Zhang D et al 2010 Rev. Sci. Instrum.81 10E134

[30] Biel W, Bertschinger G, Burhenn R, König R and Jourdain E 2004 Rev. Sci. Instrum. 753268

[31] Wenzel U et al 2018 Nucl. Fusion 58096025

[32] Tokar' M Z 1993 Plasma Phys. Controlled Fusion 351119

[33] Zhang D et al 2016 43rd EPS Conf. on Plasma Physics P4.015

[34] Svensson J 2011 JET Internal Report EFDA-JET-PR(11)24

[35] Svensson J and Werner A 2007 IEEE Int. Symp. on Intelligent Signal Processing pp 1-6

[36] Brezinsek S et al 2011 Nucl. Fusion 51073007

[37] Brezinsek S, Jachmich S, Rapp J, Meigs A G, Nicholas C, O’Mullane M and Pospieszczyk A $2011 \mathrm{~J}$. Nucl. Mater. 417 624-628 